\title{
The ETS Protein MEF Is Regulated by Phosphorylation-Dependent Proteolysis via the Protein-Ubiquitin Ligase $\mathrm{SCF}^{\mathrm{Skp} 2}$
}

\author{
Yan Liu, ${ }^{1}$ Cyrus V. Hedvat, ${ }^{1,3}$ Shifeng Mao, ${ }^{1}$ Xin-Hua Zhu, ${ }^{2}$ Jinjuan Yao, ${ }^{3}$ Hoang Nguyen, ${ }^{2}$ \\ Andrew Koff, ${ }^{2}$ and Stephen D. Nimer ${ }^{1,4 *}$ \\ Laboratory of Molecular Aspects of Hematopoiesis ${ }^{1}$ and Molecular Biology Program, ${ }^{2}$ Sloan-Kettering Institute, \\ and Department of Pathology ${ }^{3}$ and Division of Hematologic Oncology, Department of Medicine, ${ }^{4}$ \\ Memorial Sloan-Kettering Cancer Center, New York, New York 10021
}

Received 14 September 2005/Returned for modification 14 October 2005/Accepted 17 January 2006

\begin{abstract}
MEF is an ETS-related transcription factor with strong transcriptional activating activity that affects hematopoietic stem cell behavior and is required for normal NK cell and NK T-cell development. The MEF (also known as ELF4) gene is repressed by several leukemia-associated fusion transcription factor proteins (PML-retinoic acid receptor $\alpha$ and AML1-ETO), but it is also activated by retroviral insertion in several cancer models. We have previously shown that cyclin A-dependent phosphorylation of MEF largely restricts its activity to the $G_{1}$ phase of the cell cycle; we now show that MEF is a short-lived protein whose expression level also peaks during late $G_{1}$ phase. Mutagenesis studies show that the rapid turnover of MEF in $S$ phase is dependent on the specific phosphorylation of threonine 643 and serine 648 at the $C$ terminus of MEF by cdk2 and on the Skp1/Cul1/F-box (SCF) E3 ubiquitin ligase complex $\mathrm{SCF}^{\mathrm{Skp} 2}$, which targets MEF for ubiquitination and proteolysis. Overexpression of MEF drives cells through the $G_{1} / S$ transition, thereby promoting cell proliferation. The tight regulation of MEF levels during the cell cycle contributes to its effects on regulating cell cycle entry and cell proliferation.
\end{abstract}

MEF (also known as ELF4) is a member of the ETS family of transcriptional regulators (33) that was originally isolated from a human megakaryocytic leukemia cell line (23). MEF is highly homologous to ELF-1 and to NERF-1 and -2, especially in the ETS domain, which suggests that these proteins may recognize similar DNA regulatory sequences (33). MEF is a more potent transcriptional activator than ELF-1 on many promoters (12), yet it can repress transcription as well (32). The MEF (ELF4) gene is repressed by several leukemia-associated fusion transcription factor proteins (PML-retinoic acid receptor $\alpha$ and AML1-ETO), but it is also activated by retroviral insertion in several cancer models $(21,22,30)$. Analysis of MEF-null mice has shown that MEF is required for normal NK cell and NK T-cell development (20) and plays a nonredundant role in regulating hematopoietic stem cell quiescence (20a).

Several ETS proteins, such as PU.1 and ELF-1, have been shown to bind to the retinoblastoma protein $(\mathrm{Rb})$ via an LXCXE motif (40), which may allow for the cell cycle-dependent regulation of their function. We have shown that MEF binds to and is phosphorylated by cyclin $\mathrm{A}$, which reduces its transactivation of gene expression (24). Phosphorylation of cellular proteins can activate their function, change their intracellular localization, and trigger their degradation, a process which often occurs via the ubiquitin (Ub)-proteasome pathway. The level of several cell cycle regulatory proteins (such as the cyclin dependent kinase [CDK] inhibitor protein p27) and the E2F-Rb transcription factor complex are regulated by

\footnotetext{
* Corresponding author. Mailing address: Division of Hematologic Oncology, Department of Medicine, Memorial Sloan-Kettering Cancer Center, 1275 York Ave., Box 575, New York, NY 10021. Phone: (212) 639-7198. Fax: (212) 794-5849. E-mail: nimers@mskcc.org.
}

ubiquitination and proteasome-mediated degradation $(3,25)$. CDKs regulate the activity of several transcription factors, but the best-studied example is cyclin D-dependent kinase regulation of E2F function, via phosphorylation of Rb (9). Similarly, NF-кB-dependent cell survival signals are regulated by phosphorylation (of ІкB by ІкB kinase), which triggers ІкB ubiquitination and degradation, releasing NF- $\mathrm{KB}$ to enter the nucleus and turn on gene expression (29).

Ub-dependent proteolysis by the proteasome is a common regulatory mechanism for a growing number of proteins, especially those involved in cell cycle control. A class of E3 ligases, known as Skp1-Cul1/Cdc53-F-box protein (SCF) complexes, recognizes and polyubiquitinates substrates that are phosphorylated at specific sites. Roc1, Cul1, and Skp1 are the invariant core components of SCF complexes, with one of several F-box proteins imparting substrate recognition and specificity $(1,7,14,19)$. Specific SCF complexes polyubiquitinate $\mathrm{I}-\mathrm{k} \mathrm{B}\left(\mathrm{SCF}{ }^{\mathrm{BTRCP}}\right), \mathrm{p} 27^{\mathrm{Kip} 1}, \mathrm{p} 57^{\mathrm{kip} 2}$ and $\mathrm{p} 130\left(\mathrm{SCF}^{\mathrm{Skp} 2}\right)$, and cyclin $\mathrm{E}\left(\mathrm{SCF}^{\mathrm{cdc} 4}\right)$, targeting them for proteasome-mediated degradation $(6,15,17,25,35,37,39,41,42)$.

Recently, we have observed increased stem cell quiescence in the absence of MEF (20a) and more rapid cell growth when MEF is overexpressed (J. Yao et al., unpublished data). MEF activity peaked during the $\mathrm{G}_{1}$ phase of the cell cycle in a prior study (24), which led us to examine whether MEF protein levels are similarly regulated during the cell cycle.

We find that MEF is a short-lived protein whose expression decreases dramatically at the $G_{1} / S$ boundary. The half-life of $\mathrm{MEF}$ is regulated by phosphorylation at critical C-terminal serine or threonine residues, and serine 648 appears to be the key target of cyclin A1/Cdk2. Furthermore, we show that cell cycle-related phosphorylation events trigger the ubiquitination of MEF and that $\mathrm{SCF}^{\mathrm{Skp} 2}$ is the relevant protein-Ub E3 ligase, 
as Skp2 overexpression decreases MEF levels and dominant negative forms of Skp2 prolongs its half-life. The ubiquitination of MEF by $\mathrm{SCF}^{\mathrm{Skp} 2}$ is possible only after MEF is phosphorylated by cyclin $\mathrm{A} 1 / \mathrm{Cdk} 2$, and our in vitro degradation assays suggest that multiple phosphorylation events are needed to trigger the degradation of MEF. MEF plays a key role in cell cycle regulation and hematopoietic cell behavior; its function is regulated by a complex series of posttranslational, cell cycledependent modifications.

\section{MATERIALS AND METHODS}

Site-directed mutagenesis and construction of expression plasmids. Mutan human MEF cDNAs were generated by a Quick-Change site-directed mutagenesis kit (Stratagene). Primers for the phosphorylation site mutations include the following: serine 641 to alanine, 5'-AGCCTTCTGACAAGAGCCCCCACC-3'; threonine 643 to alanine, $5^{\prime}$-CCCGCCCCAGCCCCTTTCTCCCCATTCAAC$3^{\prime}$; and serine 648 to alanine, 5'-GCCCCTTTCGCCCCATTCAACCCTACTT CC- $3^{\prime}$. All mutants were confirmed by DNA sequencing and subcloned into the pCMV5 promoter-based expression plasmid.

Cell manipulations and centrifugal elutriation. SKOV3 cells and NB4 cells were grown in RPMI medium, and 293T cells were grown in Dulbecco's modified Eagle's medium, both supplemented with $10 \%$ fetal bovine serum, $2 \mathrm{mM}$ glutamine, and $100 \mathrm{U} / \mathrm{ml}$ penicillin and streptomycin (GIBCO). Cells were treated with $2 \mathrm{mM}$ hydroxyurea for $24 \mathrm{~h}$ to synchronize them at the $\mathrm{G}_{1} / \mathrm{S}$ transition. To synchronize cells at $\mathrm{G}_{2} / \mathrm{M}$, cells were treated with $2 \mu \mathrm{M}$ nocodazole for $12 \mathrm{~h}$. To synchronize cells at $\mathrm{S}$ phase, cells were treated with $2 \mathrm{mM}$ hydroxyurea for $24 \mathrm{~h}$, thoroughly washed, and then cultured in medium without hydroxyurea for another $3 \mathrm{~h}$. The cell cycle status of the synchronized cells was determined by staining cells with propidium iodide, followed by fluorescence-activated cell sorting analysis.

Logarithmically growing NB4 cells cultures were fractionated into distinct cell cycle phases by centrifugal elutriation in a Beckman J2-21 M centrifuge and a JE-6B rotor with a large $(40 \mathrm{ml})$ separation chamber, as previously described (18). For fluorescence-activated cell sorting analyses, aliquots of $10^{6}$ cells were fixed in ethanol and incubated for $30 \mathrm{~min}$ at $37^{\circ} \mathrm{C}$ in $0.5 \mathrm{ml}$ of staining solution $(25 \mu \mathrm{g} / \mathrm{ml}$ propidium iodide [PI] and $10 \mu \mathrm{g} / \mathrm{ml}$ RNase A in phosphate-buffered saline [PBS]). Stained cells were analyzed on a Becton-Dickinson FACScan.

Pulse-chase experiments. $293 \mathrm{~T}$ cells were transiently transfected with cytomegalovirus (CMV) promoter-based constructs expressing either MEF-hemagglutinin (HA; $20 \mu \mathrm{g}$ ) or MEF-TRI-A-HA (20 $\mu \mathrm{g}$; MEF-TRI-A contains three alanine substitutions at the $\mathrm{C}$ terminal putative cyclin $\mathrm{A} / \mathrm{Cdk} 2$ phosphorylation sites). After $36 \mathrm{~h}$, the cells were starved of methionine for $1 \mathrm{~h}$ (using Dulbecco's modified Eagle's medium lacking methionine), and then labeled with $100 \mu \mathrm{Ci} / \mathrm{ml}$ Redivue $\left[{ }^{35} \mathrm{~S}\right]$ methionine (Amersham Biosciences) for $1 \mathrm{~h}$. Cells were washed with PBS three times and then incubated in $20 \mathrm{ml}$ of medium supplemented with $25 \mu \mathrm{g} / \mathrm{ml}$ cycloheximide and $1 \mathrm{mM}$ unlabeled methionine. The cells were then washed twice in cold PBS and lysed in radioimmunoprecipitation assay buffer. Immunoprecipitations were performed using the anti-HA, 12CA5 monoclonal antibody (Roche).

Immunological reagents and procedures. Immunoprecipitations and Western blots were performed as described previously $(24,44)$, unless otherwise specified Antibodies used included the following: cyclin A (H-432; Santa Cruz), cyclin E (HE12; Santa Cruz), cdk2 (M2; Santa Cruz), HA (12CA5; Roche), FLAG (Sigma), SKP2 (GP45; Zymed), and p27 (C-19; Santa Cruz).

Use of proteasome inhibitors and detection of ubiquitin-MEF conjugates. The proteasome inhibitors, proteasome inhibitor I and MG132, were obtained from Calbiochem and dissolved in dimethyl sulfoxide (DMSO). Approximately $24 \mathrm{~h}$ after being transfected with CMV promoter-based expression plasmids, the 293T cells were treated with proteasome inhibitor I $(10 \mathrm{mM})$ or MG132 $(50 \mu \mathrm{M})$ for 6 to $8 \mathrm{~h}$, lysed (as above), and subjected to either immunoblotting or immunoprecipitation. For detection of ubiquitin-MEF conjugates, the cell extracts were immunoprecipitated using either the rabbit polyclonal anti-MEF antiserum or the mouse monoclonal anti-Ub antibody, followed by immunoblotting with either an anti-Ub antibody or an anti-MEF antiserum, respectively.

In vivo ubiquitination assays were performed by transfecting cells with $4 \mu \mathrm{g}$ of pMT107, which encodes polyhistidine-tagged Ub, together with the appropriate MEF expression plasmid. Twenty-four hours later, cells were harvested, and ubiquitinated proteins were purified by nickel-affinity chromatography as previously described (31).
In vitro kinase assay. Two microliters of $\mathrm{Sf} 9$ cellular extract containing a combination of cyclin D2 and CDK4, cyclin E and CDK2, or cyclin A1 and CDK2 was incubated with $0.2 \mu \mathrm{g}$ of glutathione transferase-Rb (GST-Rb; Santa Cruz) or $20 \mu \mathrm{l}$ of GST-MEF protein in a $30-\mu l$ kinase reaction mixture $(50 \mathrm{mM}$ HEPES-KOH, pH 7.5, 10 mM MgCl2, 1 mM dithiothreitol, 2.5 mM EGTA, 10 $\mathrm{mM}$ glycerophosphate, $0.1 \mathrm{mM}$ NaVO4, $1 \mathrm{mM} \mathrm{NaF}, 20 \mu \mathrm{M}$ lithium-ATP, and $\left.\left[{ }^{32} \mathrm{P}\right] \mathrm{ATP}\right)$ for $30 \mathrm{~min}$ at $30^{\circ} \mathrm{C}$. After the addition of $10 \mu \mathrm{l}$ of $4 \times$ sodium dodecyl sulfate (SDS) sample buffer, samples were boiled for $5 \mathrm{~min}$ at $95^{\circ} \mathrm{C}$ and separated by SDS-polyacrylamide gel electrophoresis (PAGE), and autoradiography was performed.

In vitro ubiquitination assays. The in vitro ubiquitination assay was done as described previously (44). $\left[{ }^{35} \mathrm{~S}\right]$ methionine-labeled proteins were prepared by in vitro transcription (with T7) polymerase and in vitro translation (using nucleasetreated rabbit reticulocyte lysate) as specified by the manufacturer (Promega). Radiolabeled MEF was first incubated at $30^{\circ} \mathrm{C}$ for 30 min with cyclin A1-cdk2 complexes in $40 \mathrm{mM}$ Tris- $\mathrm{HCl}$ (pH 7.6), $1 \mathrm{mM}$ ATP, $10 \mathrm{mM} \mathrm{MgCl} 2,1 \mathrm{mM}$ dithiothreitol, and $1 \mu \mathrm{M}$ okadaic acid. Phosphorylated MEF was added to the ubiquitination reaction mixture, which contains $10 \mathrm{mM}$ creatine phosphate, 0.1 $\mathrm{mg}$ of creatine kinase/ml, $1 \mu \mathrm{M}$ ubiquitin aldehyde, $1 \mathrm{mg}$ of methylated ubiquitin/ $\mathrm{ml}, 0.2 \mu \mathrm{g}$ of E1, $2 \mu \mathrm{g}$ of E2, $1 \mu \mathrm{g}$ of His-cul1/roc1, $0.6 \mu \mathrm{g}$ of His-skp1/skp2, and $0.05 \mu \mathrm{g}$ of cks1. All substrates and E3 components were supplied in the linear range when $50 \mathrm{ng}$ of cyclin A1-cdk2 was used as the kinase activity. Following incubation at $30^{\circ} \mathrm{C}$ for $90 \mathrm{~min}$, the samples were resolved and subjected to autoradiography analysis.

In vitro degradation assays. To measure the CDK-dependent degradation of $\mathrm{MEF}$, we utilized a cell-free degradation system that has been shown to faithfully replicate the in vivo degradation of p27 (27). Cell extracts were prepared from HeLa-S3 cells that were synchronized in $\mathrm{G}_{1}$ (by incubation in $2 \mu \mathrm{M}$ nocodazole [Sigma] for $12 \mathrm{~h}$ and then being cultured in medium without nocodazole for another $5 \mathrm{~h}$ ) or in $\mathrm{S}$ phase (by incubation in $2 \mathrm{mM}$ hydroxyurea [Sigma] for $24 \mathrm{~h}$ and then being cultured in medium without hydroxyurea for another $3 \mathrm{~h}$ ). To deplete the proteasome, extracts supplemented with rabbit reticulocyte lysate were centrifuged for $6 \mathrm{~h}$ at $100,000 \times g$ at $4^{\circ} \mathrm{C}$ and fractionated into supernatant and pellet. The pellets were subsequently resuspended in an equivalent volume of hypotonic buffer.

The degradation assay was performed essentially as described by Brandeis and Hunt (2) with minor modifications, using $200 \mu \mathrm{g}$ of extract supplemented with an ATP-regenerating system $(25 \mathrm{mM}$ phosphocreatine, $10 \mu \mathrm{g}$ of creatine kinase per $\mathrm{ml}$ ), $1 \mathrm{mM}$ ATP, and 1/15 volume of rabbit reticulocyte lysate (Promega) in a total volume of $20 \mu \mathrm{l}$ with $0.1 \mu \mathrm{l}$ of radiolabeled substrate. The reaction mixtures were incubated at $30^{\circ} \mathrm{C}$ for $2 \mathrm{~h}$, and the reactions were stopped by the addition of SDS sample buffer. Proteins were resolved by SDS-PAGE and detected by autoradiography.

\section{RESULTS}

MEF is a short-lived protein whose level is regulated during the cell cycle. Although MEF RNA is abundantly expressed in a variety of tissues, we observed that its protein expression is barely detectable by Western blot analysis in many cells growing in culture (23). This suggests that MEF might be a shortlived protein. To assess its half-life, we performed pulse-chase experiments in $293 \mathrm{~T}$ cells that we engineered to transiently express HA-tagged MEF. The half-life of tagged MEF is about $60 \mathrm{~min}$ (Fig. 1B), which is similar to the half-life of endogenous MEF protein (half-life of $\approx 1 \mathrm{~h}$ ) based on studies of SKOV3 cells exposed to cycloheximide (data not shown). We next examined the half-life of HA-tagged MEF-TRI-A, a full-length construct that contains alanine substitutions at all three $\mathrm{C}$ terminal putative cyclin $\mathrm{A} / \mathrm{Cdk} 2$ phosphorylation sites in MEF (S641A, T643A, and S648A) (24). The half-life of MEF-TRI-A is approximately $3 \mathrm{~h}$ (Fig. 1B), which is substantially longer than that of the wild-type MEF protein.

We previously reported that MEF transcriptional activity is highest in late $\mathrm{G}_{1}$ /early S phase (24), using an MEF-responsive reporter gene that did not directly assess MEF protein levels. To assess changes in MEF protein levels during the cell cycle, we first examined the endogenous level of MEF in NB4 cells 
A

MEF C- terminal

MEF-TRI-A C- terminal
(641-650) 5' S P T P APF S P F 3'

(641-650) 5' A P A P A P F A P F 3'

B

C



Cyclin A

Cdk2

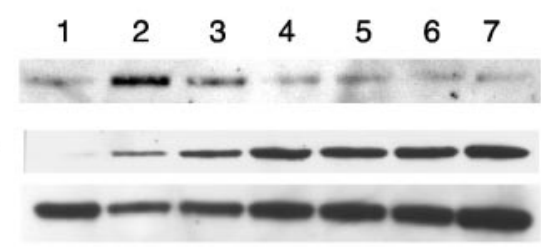

D
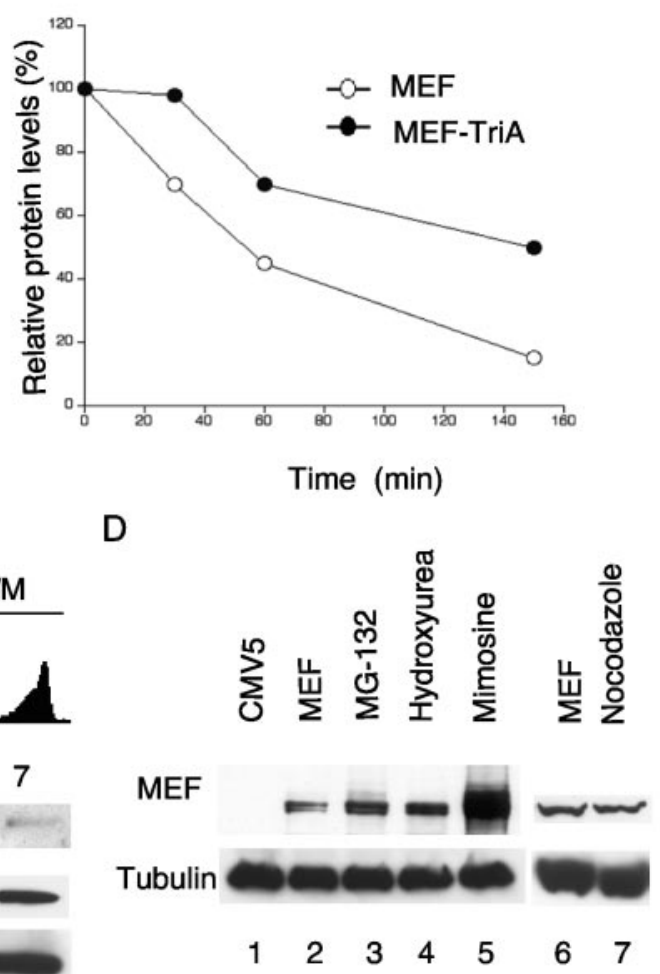

Skp2

FIG. 1. MEF is a short-lived protein whose level is regulated during the cell cycle. (A) The C-terminal amino acid sequence of MEF and the MEF-TRI-A mutant protein. (B) Pulse-chase analysis of HA-tagged wild-type MEF and HA-tagged MEF-TRI-A in 293T cells. 293T cells were transfected with pCMV5-HA-MEF or pCMV5HA-MEF-TRI-A, and $36 \mathrm{~h}$ after transfection, the cells were metabolically labeled and chased in cold medium. Lysates from the indicated time points were immunoprecipitated using an anti HA antibody and analyzed by SDS-PAGE. Quantitative measurements of band intensities by densitometry are graphed on a log scale over time (in minutes). (C) NB4 cells were separated in different stages of the cell cycle by centrifugal elutriation. The top panel shows the DNA content in each fraction, as determined by flow cytometry after PI staining. Equivalent amounts of total cell extracts were subjected to SDS-PAGE and analyzed by Western blotting with the indicated antibody. (D) Effects of the proteasome inhibitor MG132, as well as hydroxyurea, mimosine, and nocodazole on MEF levels. MEF-expressing 293T cells were treated with MG132, hydroxyurea, or mimosine for $4 \mathrm{~h}$ (lanes 3, 4, and 5) or with nocodazole or DMSO for $12 \mathrm{~h}$ (lanes 7 and 6 ). The cells were then harvested for Western blot analysis.

(an acute promyelocytic leukemia cell line) isolated at different stages of the cell cycle by centrifugal elutriation. MEF protein levels were highest during $\mathrm{G}_{1}$ (Fig. $1 \mathrm{C}$, lanes 2 and 3), decreasing significantly after the $\mathrm{G}_{1} / \mathrm{S}$ transition (Fig. $1 \mathrm{C}$, lanes 4 and 5), concurrently with the increasing expression of cyclin $A$, and reaching its lowest level during $S$ and $\mathrm{G}_{2}$ (Fig. $1 \mathrm{C}$, lanes 6 and 7). In contrast MEF RNA levels were similar in all cell fractions (not shown). Treating Kasumi-1 or SKOV-3 cells with hydroxyurea (HU) or mimosine, which block cells at the $\mathrm{G}_{1} / \mathrm{S}$ transition, results in a two- to threefold increase in the amount of MEF protein in the cell (data not shown).
Furthermore, treating 293T cells that were transiently transfected with an MEF expression plasmid with HU or mimosine also significantly increased MEF protein levels (Fig. 1D). Nocodazole, which blocks cells in $M$ phase, did not increase the level of MEF in these cells (Fig. 1D).

MEF turnover depends on proteasome function. The cell cycle regulatory protein p27 is degraded by the ubiquitin-proteasome degradation pathway (5). Similarly, a $4 \mathrm{~h}$ exposure of 293T/MEF cells to the proteasome inhibitor MG132 significantly increased MEF levels (Fig. 1D). To better define the role of this pathway in regulating MEF levels, we examined 
DMSO





\section{$\mathrm{CHX}(0.25 \mathrm{mM})$ MEF}

Tubulin

FIG. 2. MEF turnover is proteasome dependent. Thymidine-arrested NIH 3T3-MEF cells were treated with either the proteasome inhibitor MG132 or DMSO. The protein synthesis inhibitor cycloheximide $(\mathrm{CHX})$ was added after $4 \mathrm{~h}$ of treatment, and cells were harvested for Western blot analysis at the indicated times.

the effects of the proteasome inhibitor MG132 on thymidine-arrested MEF-expressing NIH 3T3 cells. MG132 treatment increased the level of MEF significantly, compared to the DMSO-treated cells (Fig. 2A), showing that MEF is degraded mainly through ubiquitin-proteasome-mediated pathways.

MEF is polyubiquitinated at the $\mathbf{G}_{\mathbf{1}} / \mathbf{S}$ transition. To determine whether MEF is ubiquitinated in vivo, we synchronized MEF-expressing $293 \mathrm{~T}$ cells in the $\mathrm{G}_{1} / \mathrm{S}$ phase using mimosine and looked for polyubiquitin-MEF conjugates using an anti-Ub antibody that specifically recognizes the 76-amino-acid ubiquitin polypeptide. Mimosine treatment resulted in a characteristic laddering of MEF-polyubiquitin (Fig. 3A, lane 3), whereas no significant laddering was seen in the absence of mimosine treatment (Fig. 3A, lane 2), likely due to the efficient degradation of MEF once it is ubiquitinated during $\mathrm{S}$ phase. Furthermore, MEF was also detected among the ubiquitinated proteins immunoprecipitated from cells treated with mimosine (Fig. 3A, lane 3) but not from untreated cells, using the anti$\mathrm{Ub}$ antibody. This demonstrates that MEF is ubiquitinated in vivo and implies that ubiquitination of MEF occurs near the $\mathrm{G}_{1} / \mathrm{S}$ transition.

To examine whether phosphorylation of MEF at its C-terminal serine or threonine residues regulates MEF ubiquitination in vivo, we used a polyhistidine-tagged Ub-based ubiquitination detection system. While MEF as well as the MEF-S641A and MEF-T643A mutant proteins were efficiently ubiquitinated, neither MEF-TRI-A nor the MEF-S648A mutant was efficiently ubiquitinated (Fig. 3B). Thus, serine phosphorylation at S648 in MEF is required for its ubiquitination; we next explored how this amino acid substitution affected the half-life of MEF protein.

MEF turnover is regulated by phosphorylation. We examined the half-life of a series of MEF single-phosphorylationsite mutants in $293 \mathrm{~T}$ cells to further define how the phosphorylation of MEF regulates its half-life. The MEF-S641A, MEF-T643A and MEF-S648A phosphorylation-site mutants had longer half-lives than MEF in cells treated with cycloheximide, with the S648A mutant protein being the most stable, followed by the T643A and then the S641A mutant proteins. Yet none of the MEF single-site mutants was as stable as MEF-TRI-A, whose level did not change during the 4-h cycloheximide treatment (Fig. 4A). This suggests that multiple phosphorylation events regulate the half-life and the degradation of MEF.

We previously reported that phosphorylation of MEF by cyclin $\mathrm{A} / \mathrm{Cdk} 2$ kinase occurs at one of the three $\mathrm{S} / \mathrm{P}$ or $\mathrm{T} / \mathrm{P}$ sites
A

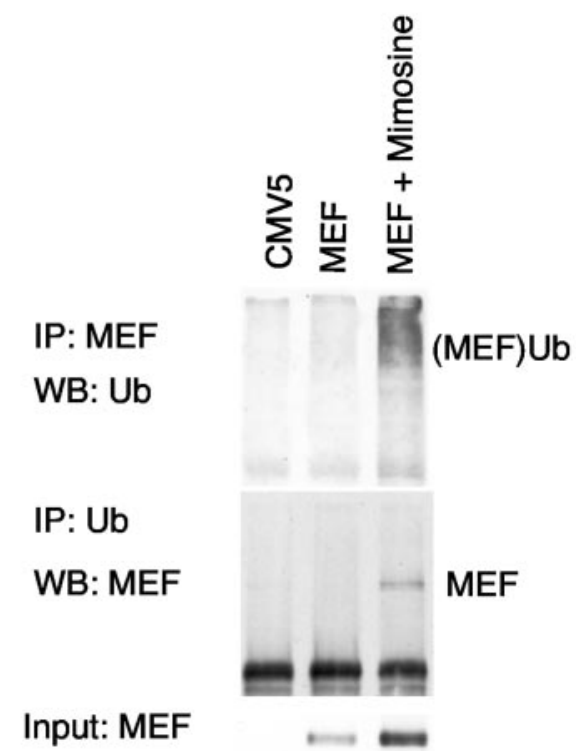

B
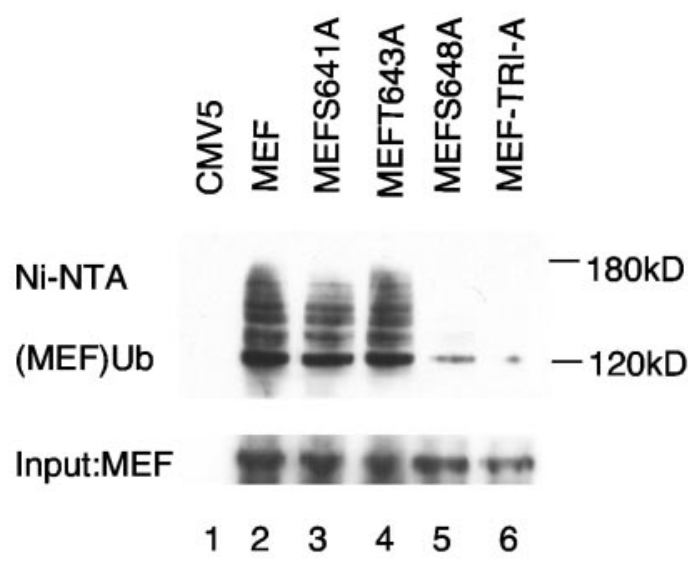

FIG. 3. Phosphorylation-dependent MEF ubiquitination. (A) $293 \mathrm{~T}$ cells were transfected with pCMV5MEF, and $24 \mathrm{~h}$ after transfection, the cells were treated with mimosine $(400 \mu \mathrm{M})$ for $6 \mathrm{~h}$. The cell lysates were subjected to immunoprecipitation (IP) using an anti-MEF antibody and then immunoblotted using an anti-ubiquitin antibody. (B) 293T cells were cotransfected with expression constructs for wild-type MEF (lane 2) or the indicated MEF phosphorylation mutants (lanes 3 to 6) and for polyhistidine-tagged Ub. His-Ub conjugates were purified and immunoblotted using an anti-MEF antibody (top blot). Input samples, taken prior to purification, were also immunoblotted with an anti-MEF antibody (bottom blot). 
A



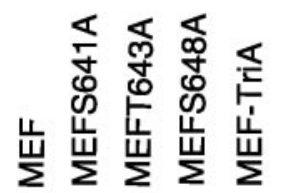

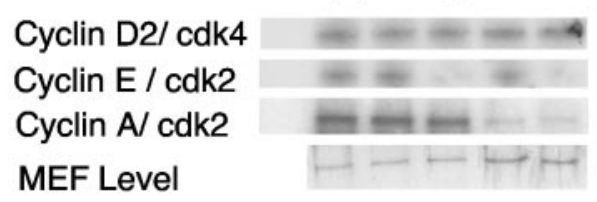

$\begin{array}{llllll}1 & 2 & 3 & 4 & 5\end{array}$

FIG. 4. MEF turnover is regulated by phosphorylation. (A) 293T cells were transfected with CMV5MEF, CMV5MEF-S641A, CMV5MEFT643A, CMV5MEF-S648A, or CMV5MEF-TRI-A. Forty-eight hours after transfection, the cells were treated with cycloheximide (0.25 mM) for the indicated time. The MEF protein level was monitored by Western blot analysis. (B) In vitro kinase assays using mutant MEF proteins. Bacterially expressed GST-MEF, GST-MEF-S641A, GST-MEF-T643A, GST-MEF-S648A, and GST-MEF-TRI-A proteins were incubated with purified cyclin D2/cdk4, cyclin E/cdk2, and cyclin A1/cdk2 recombinant proteins. Equal loading of MEF and MEF mutant proteins is shown by Coomassie blue staining. (C) Thymidine-arrested NIH 3T3-MEF cells were treated with either the CDK inhibitor roscovitine or DMSO. The protein-synthesis inhibitor cycloheximide was added after $4 \mathrm{~h}$ of treatment, and cells were harvested for Western blot analysis after the indicated time. CHX, cycloheximide.

in its $\mathrm{C}$ terminus (24). We have performed additional in vitro kinase assays using significantly purified cyclin $\mathrm{D} 2 / \mathrm{Cdk} 4$, cyclin $\mathrm{E} / \mathrm{Cdk} 2$, and cyclin $\mathrm{A} 1 / \mathrm{Cdk} 2$ to identify their preferred phosphorylation sites in MEF. Serine 648 appears to be the preferred phosphorylation site of cyclin $\mathrm{A} 1 / \mathrm{Cdk} 2$, whereas threonine 643 is the preferred phosphorylation site of cyclin E/Cdk2 (Fig. 4B). Cyclin D/Cdk4 was able to phosphorylate all of the single $\mathrm{S}$ or $\mathrm{T}$ to $\mathrm{A}$ mutants as well as MEF-TRI-A, suggesting that its preferred site is not at the $\mathrm{C}$-terminal $\mathrm{S} / \mathrm{T}$ residues. Nonetheless, these results suggest that multiple phosphorylations of MEF by cyclin-dependent kinases during late $\mathrm{G}_{1} / \mathrm{S}$ cell cycle may mediate its ubiquitination and degradation.

To establish a causal relationship between Cdk2-dependent phosphorylation and the degradation of MEF, we used the Cdk1/Cdk2-specific inhibitor roscovitine. To exclude cell cycle effects of roscovitine, we first arrested MEF-expressing $\mathrm{NIH}$
3 T3 cells in early S phase using a double-thymidine block. Cells were incubated with roscovitine for $4 \mathrm{~h}$ and then treated with cycloheximide for various lengths of time before harvesting. Using this system, roscovitine treatment stabilized the level of MEF, indicating that the degradation of MEF depends on Cdk2 activity (Fig. 4C).

MEF is a target of ubiquitylation by $\mathbf{S C F}^{\text {Skp2 }}$ complex. The SCF protein complex regulates phosphorylation-dependent proteolytic events that drive cells through the $G_{1} / S$ transition. SCF is composed of the Skp1, Cul1, and Rbx1 proteins, as well as a variable component F-box protein, which provides substrate specificity $(4,7)$. This complex functions as an E3 ligase, attaching ubiquitin molecules to the final substrate.

To address whether an SCF complex mediates the phosphorylation-dependent ubiquitination of MEF, we expressed a dominant negative version of Cul1, Cul1DN, which binds to Skp1 
A

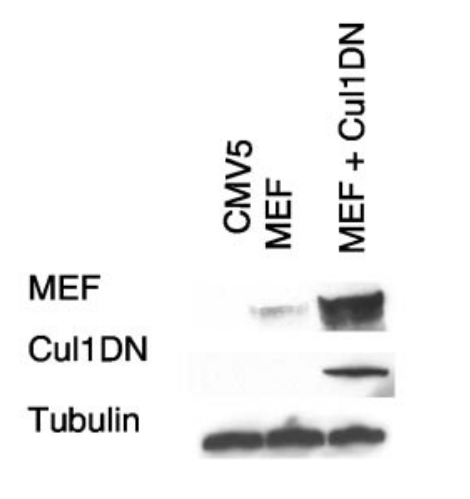

B

IP

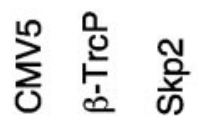

WB MEF

Skp2 input

MEF input

Tubulin

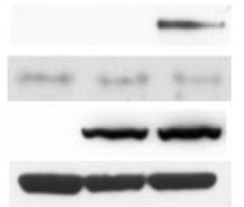

C

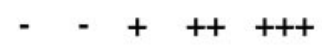

Skp2<smiles>[R16][18OH]</smiles>

$180 \mathrm{kD}-$

Ni-NTA $120 \mathrm{kD}--\cdot$

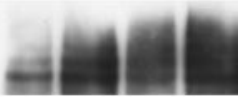

(MEF)Ub

Input / MEF

Input / Skp2

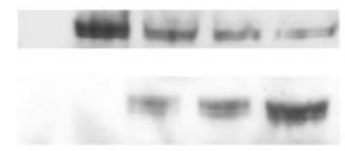

$\begin{array}{lllll}1 & 2 & 3 & 4 & 5\end{array}$
D

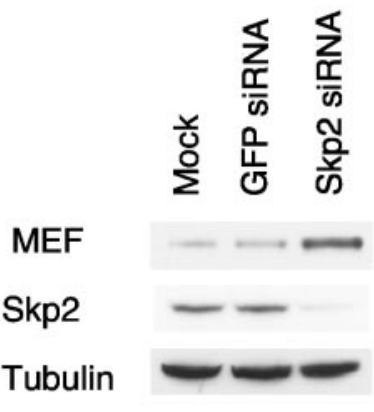

E

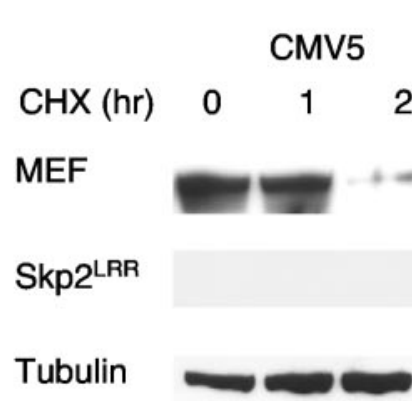

\begin{abstract}
45
\end{abstract}

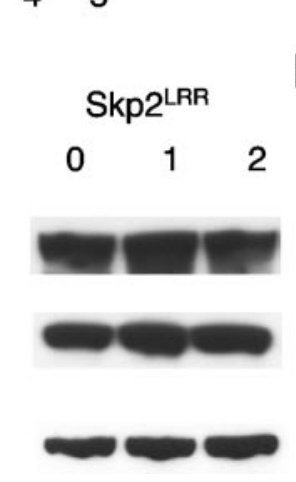

F

FIG. 5. The $\mathrm{SCF}^{\mathrm{Skp} 2}$ complex is involved in MEF ubiquitination. (A) Disruption of the Cul1 ubiquitin ligase pathway leads to accumulation of MEF. 293T cells were transfected with an MEF expression vector and a vector expressing an N-terminal fragment of Cul1 (residues 1 to 452 ; Cul1DN) or an empty vector; $48 \mathrm{~h}$ after the transfection, lysates were subjected to immunoblotting with the indicated antibodies. (B) MEF physically interacts with F-box protein Skp2. Immunoprecipitation was performed using an anti-Skp2 antibody or an anti- $\beta$-Trcp antibody and 293T-MEF cell extracts, followed by immunoblotting (WB, Western blotting) with an anti-MEF antibody. (C) Skp2 promotes the ubiquitination of MEF in vivo. 293T cells were transiently transfected with $1 \mu \mathrm{g}$ of the pCMV5MEF expression plasmid (lanes 2 to 5) and $0.5 \mu \mathrm{g}$ of the pMT107 (expressing His-tagged Ub) (lanes 2 to 5) in the absence or presence of increasing amounts (0.5, 1.0, and $2 \mu \mathrm{g}$ ) of T7-Skp2 (lanes 3 to 5). After $24 \mathrm{~h}$, the cells were harvested and lysed and put over a nickel column (top). An aliquot was kept for the analysis of the input levels of MEF (middle) and Skp2 (bottom). (D). Silencing of Skp2 expression results in stabilization of MEF. CAOV3 cells were transfected one or two times (every $24 \mathrm{~h}$ ) with siRNA molecules directed against Skp2 (or green fluorescent protein as a control). Cells were harvested and lysed $24 \mathrm{~h}$ after the last transfection, and the levels of MEF, Skp2, and tubulin in total cell extracts were analyzed by immunoblotting. (E) Inhibition of Skp2 stabilizes MEF. 293T cells were transfected with pCMV5MEF in the absence or the presence of Skp2 ${ }^{\mathrm{LRR}}$, a plasmid that expresses a dominant-negative form of Skp2. The protein synthesis inhibitor cycloheximide (CHX) was added, and the cells were harvested for Western blot analysis after the indicated time. (F) In vitro ubiquitination of MEF by the $\mathrm{SCF}^{\mathrm{Skp} 2}$ complex. In vitro ubiquitination assays were performed using reconstituted SCF ${ }^{\mathrm{kp} 2}$ complex (lanes 2 to 4 ) and cyclin A/Cdk2 with and without Cks1. In vitro translated MEF was used as the substrate for each combination. GFP, green fluorescent protein; NTA, nitrilotriacetic acid.

but does not associate with the essential RING finger protein Rbs1 (8) in 293T cells and examined its influence on MEF turnover. While the expression of Cul1DN had a minimal effect on the cell cycle distribution of these cells (by PI staining; data not shown), we found a significant accumulation of MEF
(Fig. 5A), as well as upregulation of other SCF substrates including p27 and cyclin E (data not shown). The dramatic stabilization of MEF by Cul1DN does not appear to be caused by indirect effects on cell cycle. Rather, the SCF pathway is involved in the (ubiquitination and) degradation of MEF. 
A

Cell extracts $\quad$ G1 $\quad$ S

Incubation time $(\mathrm{min}) \quad 0 \quad 30150 \quad 0 \quad 30 \quad 150$

MEF

Cyclin B1

p27
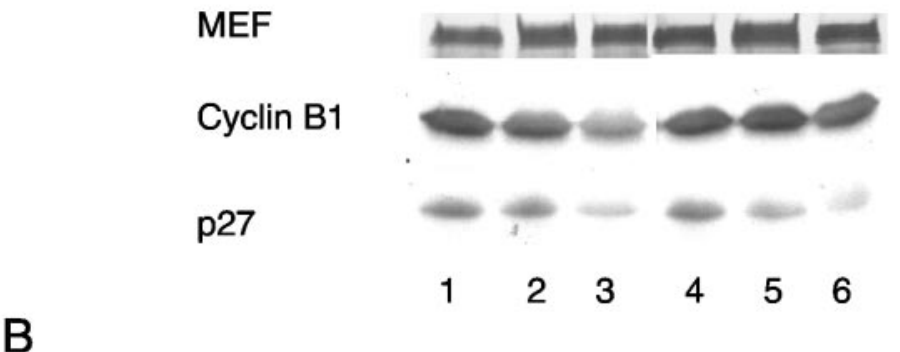

B

Cell extracts

Incubation time(hr)
G1 $\mathrm{Hu} S$

$\begin{array}{llllll}0 & 2 & 0 & 2 & 0 & 2\end{array}$

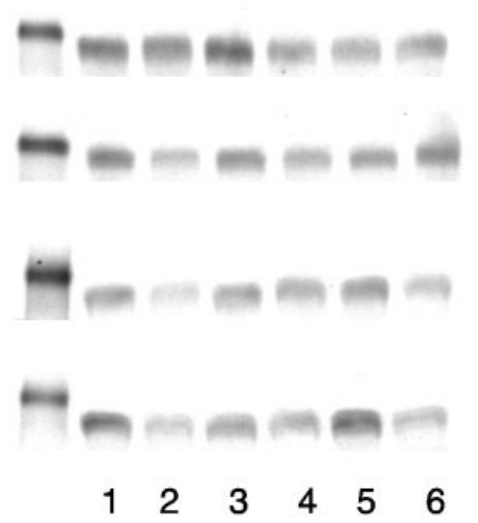

Pretreatment of

MEF with

Cyclin D1/ CDK6

Cyclin E/ CDK2

Cyclin A/ CDK2

Cyclin D1/ CDK6

+ Cyclin A/ CDK2
Cell extracts

Incubation time(hr)

\section{MEF}

MEF-Tri-A

p27

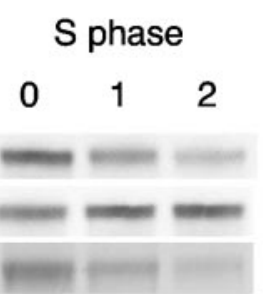

FIG. 6. In vitro degradation of MEF is most efficient following phosphorylation. (A) In vitro translated ${ }^{35}$ S-labeled MEF, cyclin B, or p27 proteins were treated with $\mathrm{G}_{1}$ or $\mathrm{S}$ phase extracts of HeLa cells for the indicated times, and protein levels were assayed by autoradiography. (B) In vitro translated MEF was pretreated with cyclin D1/cdk6, cyclin E/cdk2, or cyclin A1/cdk2 and then incubated with the $\mathrm{G}_{1}, \mathrm{G}_{1} / \mathrm{S}$, or $\mathrm{S}$ phase $\mathrm{HeLa}$ cell extracts for 0 or $2 \mathrm{~h}$. HU was used to arrest cells at $\mathrm{G}_{1} / \mathrm{S}$. The first lane shows equal amounts of MEF protein prior to incubation with extracts. (C) In vitro translated MEF, MEF-TRI-A, and p27 proteins were pretreated with cyclin A/cdk2 and then subjected to treatment with S phase extracts of HeLa cells for the indicated times. The reactions were terminated by the addition of SDS sample buffer. Proteins were resolved by SDS-PAGE and detected by autoradiography.

The substrate specificity of SCF-driven ubiquitylation reactions is controlled by the identity of the F-box protein (1), so we looked for F-box proteins that physically interact with MEF and found that Skp2, Fbx4, and Fbx7 all interact with MEF in vivo (data not shown). Skp2 was originally identified as a protein that interacts with the complex of cyclin A and Cdk2 (43), and we have shown that MEF associates with the cyclin $\mathrm{A} / \mathrm{Cdk} 2$ complex in vivo (24). Therefore, we first confirmed the MEF-Skp2 interaction by showing the binding of MEF to en- dogenous Skp2 protein in 293 T cells (Fig. 5B). Skp2 appears to be an active participant in MEF ubiquitination, as Skp2 expression in $293 \mathrm{~T}$ cells led to a dose-dependent increase in ubiquitinated MEF (Fig. 5C). Reducing the steady-state levels of Skp2 to less than $20 \%$ of normal, using small interfering RNA (siRNA) directed against Skp2, led to an accumulation of MEF protein in CAOV3 cells compared to control cells transfected with siRNAs directed against green fluorescent protein (Fig. 5D). Furthermore, expression of Skp2 ${ }^{\mathrm{LRR}}$, a 
A
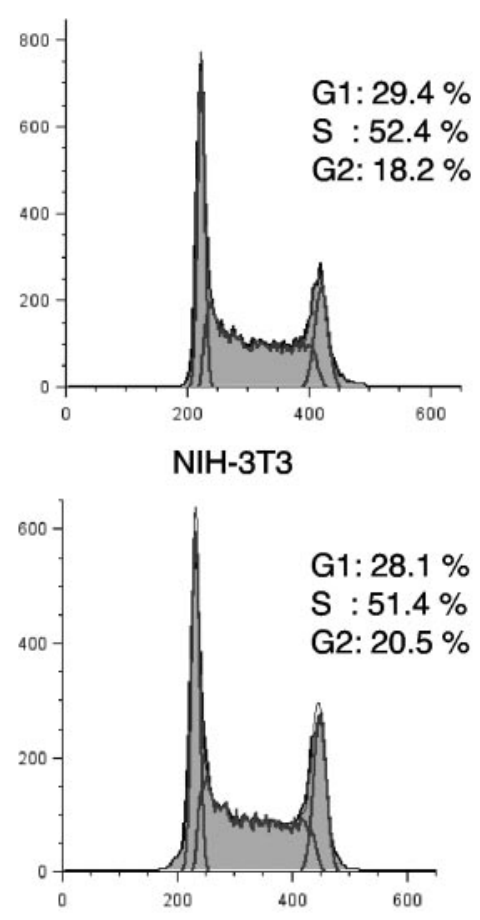

NIH-3T3 / MEF-TriA
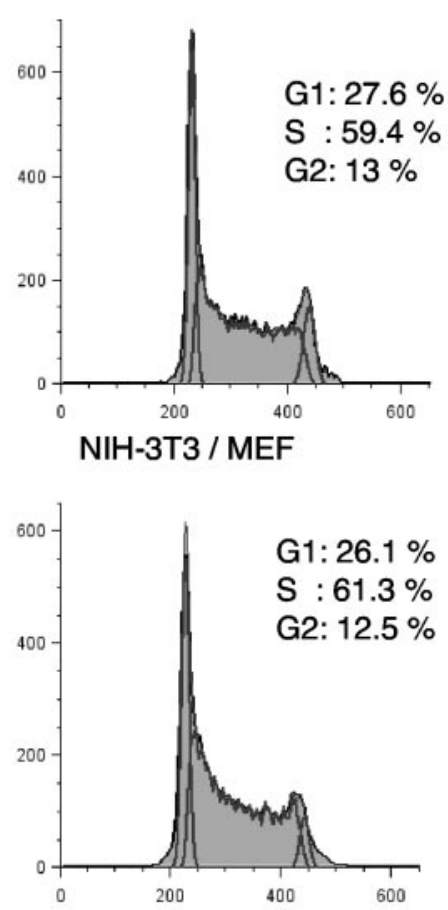

NIH-3T3 / BCR-ABL

B

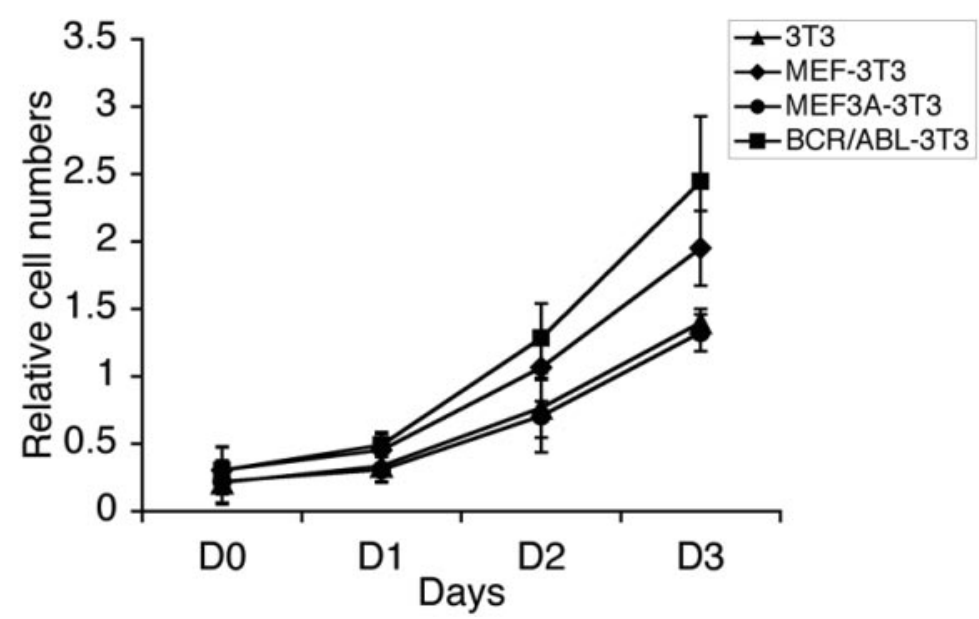

FIG. 7. Overexpression of MEF promotes S phase entry and cell proliferation. (A) Effects of MEF on cell cycle progression. NIH 3T3 cells expressing MEF, MEF-TRI-A, or BCR-ABL were subjected to flow cytometry analysis after staining with PI. The cell cycle analysis was repeated three times, and one representative result is shown. (B) Effect of MEF on cell proliferation. NIH 3 T3 cells expressing MEF, MEF-TRI-A, or BCR-ABL were subjected to proliferation assays using the WST-1 reagent. The proliferation assays were repeated three times.

dominant negative mutant form of Skp2, greatly prolonged the half-life of MEF in 293T cells (Fig. 5E).

Lastly, we examined whether an $\mathrm{SCF}^{\mathrm{Skp} 2}$ complex, assembled in vitro from purified proteins, could ubiquitinate MEF. Phosphorylated (by cyclin A1/Cdk2) but not unphosphorylated $\mathrm{MEF}$ was ubiquitinated by $\mathrm{SCF}^{\mathrm{Skp} 2}$ in the presence (or absence) of Cks1, indicating that phosphorylated MEF is a substrate of $\mathrm{SCF}^{\mathrm{Skp} 2} \mathrm{E} 3$ ubiquitin ligase and that Cks1 is not limiting in this system (Fig. 5F).

In vitro degradation of MEF depends upon sequential phosphorylation. To further define how the phosphorylation of
MEF targets it for ubiquitin-proteasome-mediated degradation, we used an in vitro proteasome-dependent protein degradation assay that we previously used to study p27 protein degradation (27). Although the $\mathrm{G}_{1}$ HeLa cell extracts efficiently degrade cyclin B1 and the S phase HeLa cell extracts degrade $\mathrm{p} 27$, neither extract degraded ${ }^{35} \mathrm{~S}-\mathrm{MEF}$, even after a 150-min incubation (Fig. 6A). We next pretreated in vitro translated MEF with several cyclin/cdk active complexes to determine whether in vitro phosphorylation can prepare MEF for efficient degradation by cell cycle-specific HeLa cell extracts. Pretreatment of MEF with cyclin $\mathrm{A} 1 / \mathrm{cdk} 2$ or cyclin 
E/cdk2 led to efficient degradation of MEF by the $G_{1}$ phase extracts (Fig. 6B). Addition of cyclin D1/cdk6 did not act cooperatively with $G_{1}$ extracts, suggesting that sequential phosphorylation is required for in vitro degradation. The degradation of MEF seen when MEF was phosphorylated in vitro by cyclin D1/cdk6 and cyclin E/cdk2 (Fig. 6B), but not by cyclin D1/cdk6 alone, further supports this finding. The lack of cooperativity between cyclin D1/cdk6 and S phase extracts suggests that some critical components required for $\mathrm{MEF}$ in degradation in this assay (other than the kinases) vary during the cell cycle (perhaps even a phosphatase).

To show that MEF is the substrate for these critical phosphorylation events, we compared the degradation of MEFTRI-A and wild-type MEF by the S phase HeLa cell extracts after cyclin A1/cdk2 pretreatment (Fig. 6C). While the extracts efficiently degrade cyclin A1/cdk2-pretreated MEF (and p27), they do not degrade MEF-TRI-A (Fig. 6C).

MEF promotes $S$ phase entry and cell proliferation. Given the peak MEF levels and activity at $\mathrm{G}_{1} / \mathrm{S}$ and the rapid fall of MEF activity at subsequent phases, we examined the effects of MEF on cell cycle events and cell proliferation. We generated NIH 3 T3 cells that stably express MEF, MEF-TRI-A, or BCRABL by retroviral transduction and performed cell cycle analysis by PI staining and flow cytometry (Fig. 7A). Overexpression of MEF increased the percentage of cells in $\mathrm{S}$ phase to a similar degree as the constitutively active BCR-ABL tyrosine kinase. MEF also stimulated the proliferation of these cells but not as potently as BCR-ABL. In contrast, the MEF-TRI-A mutant had no effect on cell cycle distribution or cell proliferation (Fig. 7B), implying that phosphorylation or ubiquitination of MEF is essential for it to promote cell cycle progression and cellular proliferation.

\section{DISCUSSION}

The proteins that regulate the cell cycle are themselves regulated by a series of transcriptional and posttranslational mechanisms, including phosphorylation, acetylation, and ubiquitylation, which can alter protein function. The role that cyclin gene expression and cdk inhibitor functional regulation plays in cell cycle events has been extensively elucidated, and while the $\mathrm{E} 2 \mathrm{~F} / \mathrm{Rb}$ pathway has been most extensively investigated, it is clear that other factors contribute to appropriate cell cycle control.

Ets family (ETS) transcription factors play important roles in cell development and in cellular differentiation, proliferation, and apoptosis. The aberrant expression of ETS genes has been observed in various types of malignancies $(28,33)$. ETS proteins such as ELF-1, PU.1, and MEF may also be regulated in a cell cycle-dependent manner. Both PU.1 and ELF-1 bind $\mathrm{Rb}(10,40)$, although changes in their activity during the cell cycle have not been well documented. Here we show that MEF protein levels are controlled in a cell cycle-dependent, phosphorylation-dependent, and $\mathrm{SCF}^{\mathrm{Skp} 2}$-dependent manner.

MEF is a short-lived protein, whose activity peaks in $G_{1}$ phase. We have previously established that MEF is a substrate for the cyclin $\mathrm{A} / \mathrm{cdk} 2$ complex, yet phosphorylation by cyclin $\mathrm{A} / \mathrm{cdk} 2$ alone appears to be insufficient to trigger MEF degradation. It appears that the sequential phosphorylation of MEF by cyclin/cdk complexes (possibly first by cyclin $\mathrm{D} / \mathrm{cdk} 4$, and then by cyclin $\mathrm{E} / \mathrm{cdk} 2$ or cyclin $\mathrm{A} / \mathrm{cdk} 2$ ) triggers its ubiquitination and degradation. Consistent with the ability of cyclin A to block transactivation by MEF, the serine 648 residue appears to be the most critical for determining its stability. Our studies showed that serine 648 is the major site in MEF for phosphorylation by cyclin A1/cdk2 and for ubiquitination. Threonine 643 also contributes to the rapid turnover of MEF protein, and it seems to be the preferred site of cyclin E/cdk2 phosphorylation. Yet no single site affects cyclin A-dependent degradation of MEF as much as the TRI-A mutant does.

The ubiquitination and subsequent proteasomal degradation of regulatory proteins control a variety of cellular processes, including cell cycle progression, gene transcription, and signal transduction. E3 ubiquitin ligases have been classified into three groups: the single-subunit RING finger type, the multisubunit RING finger type, and the HECT-domain type (11). The SCF complex is a multisubunit Ub ligase that specifically transfers activated Ub to target protein substrates (4). Each F-box protein appears to be matched with a discrete number of specific substrates through a protein-protein interaction domain $(34,41)$. SCF ${ }^{\text {Skp2 }}$ complex has been shown to play a critical role in regulation of cell cycle progression by controlling the abundance of key cell cycle regulators, such as p27 $7^{\mathrm{Kip} 1}$, p5 $7^{\mathrm{Kip} 2}$, Myc, and p130 $(5,15,16,37)$. Recently, the transcription factor, FOXO1, was shown to interact with Skp2 (13). We have now provided robust evidence that Skp2 also interacts with MEF and thereby induces its Ub-dependent proteolysis. Our data also show that the degradation of MEF is not perfectly correlated with the level of ubiquitination. Other events may also control this process, as it seems that some MEF mutant proteins (such as MEF-S648A) are degraded in a Ubindependent manner (Fig. 3B and 4C).

A variety of hematopoietic and nonhematopoietic gene targets of MEF have been identified (12). Most of these are not obviously cell cycle regulated, but this may reflect the manner in which they were identified, as many genes are preferentially expressed during $\mathrm{G}_{1}$. Examination of MEF null mice provides a model system to define how MEF and the related ETS proteins ELF-1 and NERF contribute to gene expression. While NK cells are unable to express perforin in the absence of MEF, a major defect in MEF null mice is that MEF null hematopoietic stem cells have difficulty entering $\mathrm{S}$ phase in response to early acting cytokines (SCF, interleukin-3, and interleukin-6). Both hematopoietic stem cell quiescence and movement into $\mathrm{S}$ phase are altered in MEF knockout mice (20a). Conversely, overexpression of MEF increases the proliferation of nonhematopoietic cell lines (J. Yao et al., unpublished data). While MEF promotes cell cycle progression and cell proliferation, the mechanisms it utilizes to do this are still unknown.

Recently, there is more evidence supporting the hypothesis that a Ub-proteasome system controls the abundance and activity, as well as the localization of transcription activators (16, $26,31,36,38)$. Posttranslational modification of MEF is likely to be critical to its effects on the cell cycle and cell proliferation, as expression of the MEF-TRI-A mutant protein (which has a longer half-life but cannot be properly phosphorylated or ubiquitinated) did not promote cell cycle progression and the growth of cells. The tight regulation of MEF during the cell 
cycle allows it to influence cell cycle events and cellular proliferation.

\section{ACKNOWLEDGMENTS}

This work was supported by National Institutes of Health grant DK 52208 (S.D.N.) and by the Herbert Friedman Cancer Research Fund. A.K. is supported by National Institutes of Health grant GM 52597 and C.V.H. is supported by NIH grant K08 HL04478.

We thank Ning Zheng and Nikola Pavletich for providing the skp1, skp2, and cks1 reagents; William P. Tansey for skp2 and skp2 $2^{\mathrm{LRR}}$ expression plasmids; Michele Pagano for providing the Cul1DN and F-box protein expression plasmids; and Ellie Park for her assistance in preparing the manuscript.

\section{REFERENCES}

1. Bai, C., P. Sen, K. Hofmann, L. Ma, M. Goebl, J. W. Harper, and S. J. Elledge. 1996. SKP1 connects cell cycle regulators to the ubiquitin proteolysis machinery through a novel motif, the F-box. Cell 86:263-274.

2. Brandeis, M., and T. Hunt. 1996. The proteolysis of mitotic cyclins in mammalian cells persists from the end of mitosis until the onset of $\mathrm{S}$ phase. EMBO J. 15:5280-5289.

3. Campanero, M. R., and E. K. Flemington. 1997. Regulation of E2F through ubiquitin-proteasome-dependent degradation: stabilization by the $\mathrm{pRB}$ tumor suppressor protein. Proc. Natl. Acad. Sci. USA 94:2221-2226.

4. Cardozo, T., and M. Pagano. 2004. The SCF ubiquitin ligase: insights into a molecular machine. Nat. Rev. Mol. Cell. Biol. 5:739-751.

5. Carrano, A. C., E. Eytan, A. Hershko, and M. Pagano. 1999. SKP2 is required for ubiquitin-mediated degradation of the CDK inhibitor p27. Nat. Cell. Biol. 1:193-199.

6. Clurman, B. E., R. J. Sheaff, K. Thress, M. Groudine, and J. M. Roberts. 1996. Turnover of cyclin $\mathrm{E}$ by the ubiquitin-proteasome pathway is regulated by cdk2 binding and cyclin phosphorylation. Genes Dev. 10:1979-1990.

7. Deshaies, R. J. 1999. SCF and cullin/ring H2-based ubiquitin ligases. Annu. Rev. Cell Dev. Biol. 15:435-467.

8. Donzelli, M., M. Squatrito, D. Ganoth, A. Hershko, M. Pagano, and G. F. Draetta. 2002. Dual mode of degradation of Cdc25 A phosphatase. EMBO J. 21:4875-4884.

9. Dynlacht, B. D., O. Flores, J. A. Lees, and E. Harlow. 1994. Differential regulation of E2F transactivation by cyclin/cdk2 complexes. Genes Dev. 8:1772-1786.

10. Hagemeier, C., A. J. Bannister, A. Cook, and T. Kouzarides. 1993. The activation domain of transcription factor PU.1 binds the retinoblastoma (RB) protein and the transcription factor TFIID in vitro: RB shows sequence similarity to TFIID and TFIIB. Proc. Natl. Acad. Sci. USA 90:1580-1584.

11. Hatakeyama, S., and K. I. Nakayama. 2003. U-box proteins as a new family of ubiquitin ligases. Biochem. Biophys. Res. Commun. 302:635-645.

12. Hedvat, C. V., J. Yao, R. A. Sokolic, and S. D. Nimer. 2004. Myeloid ELF1like factor is a potent activator of interleukin-8 expression in hematopoietic cells. J. Biol. Chem. 279:6395-6400.

13. Huang, H., K. M. Regan, F. Wang, D. Wang, D. I. Smith, J. M. van Deursen, and D. J. Tindall. 2005. Skp2 inhibits FOXO1 in tumor suppression through ubiquitin-mediated degradation. Proc. Natl. Acad. Sci. USA 102:1649-1654.

14. Jackson, P. K., A. G. Eldridge, E. Freed, L. Furstenthal, J. Y. Hsu, B. K. Kaiser, and J. D. Reimann. 2000. The lore of the RINGs: substrate recognition and catalysis by ubiquitin ligases. Trends Cell. Biol. 10:429-439.

15. Kamura, T., T. Hara, S. Kotoshiba, M. Yada, N. Ishida, H. Imaki, S. Hatakeyama, K. Nakayama, and K. I. Nakayama. 2003. Degradation of p57Kip2 mediated by SCFSkp2-dependent ubiquitylation. Proc. Natl. Acad. Sci. USA 100:10231-10236.

16. Kim, S. Y., A. Herbst, K. A. Tworkowski, S. E. Salghetti, and W. P. Tansey. 2003. Skp2 regulates Myc protein stability and activity. Mol. Cell 11:11771188.

17. Koepp, D. M., L. K. Schaefer, X., Ye, K. Keyomarsi, C. Chu, J. W. Harper, and S. J. Elledge. 2001. Phosphorylation-dependent ubiquitination of cyclin E by the SCFFbw7 ubiquitin ligase. Science 294:173-177.

18. Koff, A., A. Giordano, D., Desai, K., Yamashita, J. W. Harper, S. J. Elledge, T. Nishimoto, D. O. Morgan, B. R. Franza, and J. M. Roberts. 1992. Formation and activation of a cyclin E-cdk 2 complex during the $G_{1}$ phase of the human cell cycle. Science 257:1689-1694.

19. Krek, W. 1998. Proteolysis and the $G_{1}-S$ transition: the SCF connection. Curr. Opin. Genet. Dev. 8:36-42.

20. Lacorazza, H. D., Y. Miyazaki, A. D. Cristofano, A. Deblasio, V. Hedvat, J. Zhang, C. Cordon-Cardo, S Mao, P. P. Pandolfi, and S. D. Nimer. 2002. The
ETS protein MEF plays a critical role in perforin gene expression and the development of natural killer and NK-T cells. Immunity 17:437-449.

20a.Lacorazza, H. D., T. Yamada, Y. Liu, Y. Miyata, M. Silvina, J. Nunes, and S. D. Nimer. The transcription factor MEF/ELF4 regulates the quiescence of primitive hematopoietic cells. Cell, in press.

21. Lund, A. H., G. Turner, A. Trubetskoy, E. Verhoeven, E. Wientjens, D. Hulsman, R. Russell, R. A. DePinho, J. Lenz, and M. van Lohuizen, M. 2002 Genome-wide retroviral insertional tagging of genes involved in cancer in Cdkn2a-deficient mice. Nat. Genet. 32:160-165.

22. Mao, S., R. C. Frank, J. Zhang, Y. Miyazaki, and S. D. Nimer. 1999. Functional and physical interactions between AML1 proteins and an ETS protein, MEF: implications for the pathogenesis of $\mathrm{t}(8 ; 21)$-positive leukemias. Mol. Cell. Biol. 19:3635-3644.

23. Miyazaki, Y., X. Sun, H. Uchida, J. Zhang, and S. D. Nimer. 1996. MEF, a novel transcription factor with an Elf-1 like DNA binding domain but distinct transcriptional activating properties. Oncogene 13:1721-1729.

24. Miyazaki, Y., P. Boccuni, S. Mao, J. Zhang, H. Erdjument-Bromage, P. Tempst, H. Kiyokawa, and S. D. Nimer. 2001. Cyclin A-dependent phosphorylation of the ETS-related protein, MEF, restricts its activity to the $G_{1}$ phase of the cell cycle. J. Biol. Chem. 276:40528-40536.

25. Montagnoli, A., F. Fiore, E. Eytan, A. C. Carrano, A. C., G. F. Draetta, A. Hershko, and M. Pagano. 1999. Ubiquitination of p27 is regulated by Cdkdependent phosphorylation and trimeric complex formation. Genes Dev. 13:1881-1889.

26. Muratani, M., and W. P. Tansey. 2003. How the ubiquitin-proteasome system controls transcription. Nat. Rev. Mol. Cell. Biol. 4:1-10.

27. Nguyen, H., D. M. Gitig, and A. Koff. 1999. Cell-free degradation of p2 $7^{\mathrm{kip} 1}$ a $\mathrm{G}_{1}$ cyclin-dependent kinase inhibitor, is dependent on CDK2 activity and the proteasome. Mol. Cell. Biol. 19:1190-1201.

28. Oikawa, T. 2004. ETS transcription factors: possible targets for cancer therapy. Cancer Sci. 95:626-633.

29. Palombella, V. J., O. J. Rando, A. L. Goldberg, and T. Maniatis. 1994. The ubiquitin-proteasome pathway is required for processing the NF-B1 precursor protein and the activation of NF-B. Cell 78:773-785.

30. Park, D. J., P. T. Vuong, S. de Vos, D. Douer, and H. P. Koeffler. 2003. Comparative analysis of genes regulated by PML/RAR alpha and PLZF/ RAR alpha in response to retinoic acid using oligonucleotide arrays. Blood 102:3727-3736.

31. Salghetti, S. E, A. A. Caudy, J. G. Chenoweth, and W. P. Tansey. 2001. Regulation of transcriptional activation domain function by ubiquitin. Science 293:1651-1653.

32. Seki, Y., M. A. Suico, A. Uto, A. Hisatsune, T. Shuto, Y. Isohama, and H. Kai. 2001. The ETS transcription factor MEF is a candidate tumor suppressor gene on the X chromosome. Cancer Res. 62:6579-6586.

33. Sharrocks, A. D. 2002. The ETS-domain transcription factor family. Nat. Rev. Mol. Cell. Biol. 2:827-837.

34. Skowyra, D., K. L. Craig, M. Tyers, S. J. Elledge, and J. W. Harper. 1997. F-box proteins are receptors that recruit phosphorylated substrates to the SCF ubiquitin-ligase complex. Cell 91:209-219.

35. Strohmaier, H., C. H. Spruck, P. Kaiser, K. A. Won, O. Sangfelt, and S. I. Reed. 2001. Human F-box protein hCdc4 targets cyclin E for proteolysis and is mutated in a breast cancer cell line. Nature 413:316-322.

36. Tansey, W. P. 2001. Transcriptional activation: risky business. Genes Dev. 15:1045-1050.

37. Tedesco, D., J. Lukas, and S. I. Reed. 2002. The pRb-related protein p130 is regulated by phosphorylation-dependent proteolysis via the protein-ubiquitin ligase SCFskp2. Genes Dev. 16:2946-2957.

38. Tootle, T. L., and I. Rebay. 2005. Post-translational modifications influence transcription factor activity: a view from the ETS superfamily. Bioessays 27:285-298.

39. Vlach, J., S. Hennecke, and B. Amati. 1997. Phosphorylation-dependent degradation of the cyclin-dependent kinase inhibitor p27. EMBO J. 16:53345344 .

40. Wang, C. Y., B. Petryniak, C. B. Thompson, W. G. Kaelin, and J. M. Leiden. 1993. Regulation of the Ets-related transcription factor Elf- 1 by binding to the retinoblastoma protein. Science 260:1330-1335.

41. Winston, J. T., D. M. Koepp, C. Zhu, S. J. Elledge, and J. W. Harper. 1999. A family of mammalian F-box proteins. Curr. Biol. 9:1180-1182.

42. Won, K. A., and S. I. Reed. 1996. Activation of cyclin E/CDK2 is coupled to site-specific autophosphorylation and ubiquitin-dependent degradation of cyclin E. EMBO J. 15:4182-4193.

43. Zhang, H., R. Kobayashi, K. Galaktionov, and D. Beach. 1995. p19Skp1 and p45Skp2 are essential elements of the cyclin A-CDK2 S phase kinase. Cell 82:915-925.

44. Zhu, X. H., H. Nguyen, H. D. Halicha, F. Traganos, and A. Koff. 2004. Noncatalytic requirement for cyclin A-cdk2 in p27 turnover. Mol. Cell. Biol. 24:6058-6066. 